\title{
Low Dimensionality as a Factor Stimulating Formation of the Cooper-Like Pairs Characteristic for Superconductors
}

\author{
Stanisław Olszewski*, Tomasz Roliński \\ Institute of Physical Chemistry, Polish Academy of Sciences, Kasprzaka, Warsaw, Poland \\ E-mail:*olsz@ichf.edu.pl \\ Received August 10, 2010; revised October 1, 2010; accepted September 16, 2010
}

\begin{abstract}
The electron gas examined in a very thin potential tube exhibits some special kind of the excited pairs making them similar to the Cooper pairs. The coupling energy of the pair can be calculated as an amount of energy required to transform the excitation energy of a coupled pair into the one-electron excitation energy. For an extremely thin potential tube the coupling energy of the pair tends to infinity. The gas energy is unstable with respect to the pair excitation which provides a kind of gap near the Fermi level. A decisive part of the gap energy is due to the electron-electron interaction. The gap is attained on condition the length of a thin potential box exceeds some critical value. In the next step, a coherence length in the gas is obtained. This length, combined with a critical magnetic field representing a transition from a superconducting to a normal state, allows us to calculate the penetration depth of the magnetic field for the singlet and triplet excitations. The penetration depth together with the critical magnetic field and energy gap can provide us with a critical current, as well as critical temperature for the superconducting state.
\end{abstract}

Keywords: One-Dimensional Superconductors, Electron-Electron Interaction, Cooper-Like Pairs

\section{Introduction}

Discovery of the superconducting behaviour in the nearly one-dimensional compounds, as well as advances in semiconductor technology of the wire-like structures, provided a special impact of interest in the one-dimensional many-electron systems. One of the main features of the superconducting state described by the BardeenCooper-Schrieffer (BCS) theory is that two one-electron states are occupied simultaneously by an electron pair called the Cooper pair. The electrons in the pair have the same absolute value of momentum, but an opposite spin. Moreover, if the electron momenta are considered in the space of the wave vector $\vec{k}$, their directions in the pair are exactly opposite. This property is characteristic also for the excited states of the pair. A factor coupling the electron pair together is usually attributed to the crystal lattice: it is assumed that some phonons can provide an attractive potential between electrons putting the pair in a kind of the bound state. If some gap exists between the ground state and an excited state of the Fermi sea, the transport of the pair occupying the excited state can exhibit the properties of a superconducting behaviour.

The superconductivity in one dimension has attracted much theoretical attention [1-4] especially after the experimental discovery of superconducting effects in the one-dimensional organic conductors [5-9]. But an interest came also because of the early claim that a high temperature superconductivity can be realized in one-dimensional materials [10]. In this context an interesting indication concerning an increase of superconducting correlation due to dimensionality change in quasi-onedimensional conductors has been also done [11]. Simultaneously, the nature of supeconductivity in quasi onedimensional systems seems to remain controversial since its discovery [12].

An important remark on superconductivity in one dimension has been done by Friedel who suggested that the coupling potential characteristic for the electron pair is due to the electron-electron interaction and is not a phonon-mediated effect $[13,14]$. The purpose of the present paper is to point out that, in fact, a pair-like excitement of the electron gas typical for the Cooper pairs is not necessarily limited to a situation due to the lattice potential and an interaction provided by a phonon-like coupling. In particular, it is demonstrated that the coupling of an electron pair, and an excitement of the coupled pairs, can be an effect strictly connected 
with the low dimensionality of the electron gas. More accurately, the pairing is due to the properties of the Coulomb and exchange interaction between electrons which become sound on condition the space occupied by the electrons is narrowed to a very thin potential tube.

In the detailed calculations the Coulomb and exchange interactions in one-dimensional systems are studied in the framework of the Hartree-Fock approximation. It can be shown that these interactions can give a strong coupling energy of the electron pair having the same absolute momenta, but opposite spin, on condition the transversal cross-section of the gas potential tube is tending to zero. The calculation of the excitation energies of the one-dimensional electron gas is also based on the Hartree-Fock method.

Evidently, in a physical practice the electron gas can never become strictly one-dimensional, nevertheless this kind of situation is roughly approached when electrons are enclosed in a very long and very thin potential tube. Simultaneously, because the Pauli principle and the Fermi statistics should be obeyed also in this limiting case, the magnetic properties of the electron ensemble become here of importance. These effects are studied on the same footing as applied to the electron interactions in a non-magnetic gas.

\section{One-Electron and Two-Electron Excitation Energy of an Almost One-Dimensional Electron Gas}

The electron gas energy in the approximation by Hartree and Fock is a sum of the kinetic energy and the Coulomb and exchange energy due to the interaction between the electron particles. The interaction of electrons with a positive core can be neglected because it can be considered as a constant term which remains unchanged in course of the electron excitation process. The potential tube is assumed to be so narrow that only the kinetic energy excitations of the longitudinal motion of electrons along that tube should be taken into account, with no allowed transversal kind of the electron transitions.

In a ground state of the gas its one-electron levels are assumed to be doubly occupied by electrons of an opposite spin between the lowest level of $n=1$ and the highest occupied level $n=n_{F}$. In consequence, there exists no net magnetic moment of such a gas; see Figure 1.

An electron excitation from some level $n_{g}$ located within the interval

$$
\begin{gathered}
1 \leq n_{g} \leq n_{F} \\
\text { to a level } n_{e} \text { above } \begin{array}{l}
n_{F} \text {, so } \\
n_{e}>n_{F},
\end{array}
\end{gathered}
$$

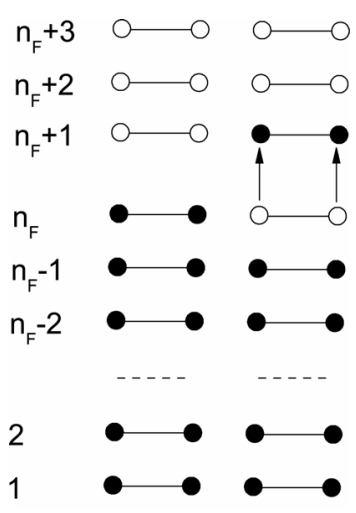

(a)

(b)

Figure 1. Pattern of the energy levels filling the Fermi sea of a non-magnetic one-dimensional electron gas: (a) a scheme before excitations, (b) a scheme after a pair excitement from the level $n_{F}$ to the level $n_{F}+1$. Full circles denote the occupied electron states.

requires an excitation of the kinetic energy by the amount (see e.g. [15])

$$
\Delta E_{k i n}=\frac{h^{2}}{8 m_{e} L^{2}}\left(n_{e}^{2}-n_{g}^{2}\right)
$$

Here $L$ is the length of the gas potential tube. Simultaneously, the electron interaction energy due to an excitation from $n_{g}$ to $n_{e}$ is changed by the amount (see e.g. [16])

$$
\begin{aligned}
& \Delta E_{e e}=\sum_{\substack{i=1 \\
i \neq n_{g}}}^{i=n_{F}=N / 2}\left[\left(2 J_{n_{e} i}-K_{n_{e} i}\right)-\left(2 J_{n_{g} i}-K_{n_{g} i}\right)\right] \\
& +J_{n_{e} n_{g}}-J_{n_{n n_{g}}} \pm K_{n_{e} n_{g}}
\end{aligned}
$$

$J$ and $K$ are the Coulomb and exchange integrals, respectively, the plus sign before $K$ in the last step of (3) refers to a singlet excited state of the gas, the minus sign - to a triplet state. In the absence of the magnetic field the quantum levels of a non-magnetic gas of $N$ electrons in their ground state are doubly occupied by electrons having opposite spin, so $n_{F}=N / 2$.

More detailed formulae are:

$$
\begin{gathered}
J_{i j}=\int d \vec{r}_{1} \int d \vec{r}_{2} \Psi_{i}^{2}\left(\vec{r}_{1}\right) \Psi_{j}^{2}\left(\vec{r}_{2}\right) \frac{e^{2}}{\left|\vec{r}_{1}-\vec{r}_{2}\right|}, \\
K_{i j}=\int d \vec{r}_{1} \int d \vec{r}_{2} \Psi_{i}\left(\vec{r}_{1}\right) \Psi_{j}\left(\vec{r}_{1}\right) \Psi_{i}\left(\vec{r}_{2}\right) \Psi_{j}\left(\vec{r}_{2}\right) \frac{e^{2}}{\left|\vec{r}_{1}-\vec{r}_{2}\right|},
\end{gathered}
$$

where

$$
\Psi_{\alpha}(\vec{r})=\left(\frac{2}{L}\right)^{1 / 2} \sin \left(\frac{n \pi}{L z}\right) N_{l q} J_{l}\left(u_{l q} r\right) \cos l \vartheta
$$

with 


$$
\alpha=\alpha(n, l, q),
$$

is a real wave function of a free electron vanishing at the boundary of the potential box. $N_{l q}$ is the normalzation coefficient of the $(r, \vartheta)$-dependent part of $\Psi_{\alpha}(\vec{r}) . J_{l}$ is the Bessel function of the first kind and index $l$ extended over the interval

$$
0<r<R,
$$

where $R$ is the radius of a circular cross-section of the cylindrical potential tube, $u_{l q}$ is the argument giving $q$ th zero of the function $J_{l}$ at $r=R$. Regularly, the radius $R$ is assumed so small that excitations along the potential tube are only considered. This means that only quantum number $n$ can be changed in course of an excitation process and the number $l=0$ as well as $u_{l q}=u_{01} \cong 2.405 R^{-1}$ remain constant for the whole of calculations.

In a thin potential tube any Coulomb integral $J_{n m}$ $(n \neq m)$ has a large component [17]

$$
-2 \frac{e^{2}}{L} \ln R,
$$

and the same component enters exchange integral $K_{n m}$ $(n \neq m)$. This implies that at $R \rightarrow 0$ the expression in (3) given by the terms enclosed within the square brackets converges. A difficulty comes from the remainder terms in (3) which are

$$
J_{n_{e} n_{g}}-J_{n_{g} n_{g}} \pm K_{n_{e} n_{g}} .
$$

Any integral $J_{n n}$ has its large component [17]

$$
-3 \frac{e^{2}}{L} \ln R
$$

instead of (8). In effect, for the plus sign before $K_{n_{e^{n}}}$ the dominant terms in expression (3a) become at small $R$ equal to:

$$
(-2+3) \frac{e^{2}}{L} \ln R+(-2) \frac{e^{2}}{L} \ln R=-\frac{e^{2}}{L} \ln R .
$$

This is a large positive number. On the other hand, for the minus sign before $K_{n_{e^{n}}}$, the expression (3a) gives the dominant terms

$$
(-2+3) \frac{e^{2}}{L} \ln R-(-2) \frac{e^{2}}{L} \ln R=3 \frac{e^{2}}{L} \ln R,
$$

which is a large negative number at small $R$.

In effect, for a plus sign before $K_{n_{e} n_{g}}$ characteristic for a singlet excited state, the expression in (3) diverges at $R \rightarrow 0$ giving

$$
\Delta E_{e e} \rightarrow \infty,
$$

which provides us with an infinite (positive) excitation energy at $R \rightarrow 0$.
But a different situation can exist, however, when not a single electron but an electron pair occupying the same one-electron level $n_{g} \leq n_{F}$ is excited to a level $n_{e}>n_{F}$, so after an excitation both electrons occupy $n_{e}$. The change of the kinetic energy associated with such transition is twice of that represented in (2):

$$
\Delta E_{k i n}=2 \frac{h^{2}}{8 m_{e} L^{2}}\left(n_{e}^{2}-n_{g}^{2}\right) .
$$

Simultaneously, the electron-electron interaction energy of an electron pair located first on level $n_{g}$, next on level $n_{e}$, is changed by the amount [16]:

$$
\Delta E_{e e}^{\text {pair }}=J_{n_{e} n_{e}}-J_{n_{g} n_{g}} .
$$

But each Coulomb integral having its both indices the same, contains the same term (8a) as its component [17]. This property makes (11) a convergent result also at $R \rightarrow 0$.

A full change of the electron-electron interaction energy of the gas due to the pair excitement from $n_{g}$ to $n_{e}$ becomes:

$$
\begin{aligned}
& \Delta E_{e e}=4 \sum_{\substack{i=1 \\
i \neq n_{g}}}^{i=n_{F}}\left(J_{i n_{e}}-J_{i n_{g}}\right)-2 \sum_{\substack{i=1 \\
i \neq n_{g}}}^{i=n_{F}}\left(K_{i n_{e}}-K_{i n_{g}}\right) \\
& +J_{n_{e} n_{e}}-J_{n_{g} n_{g}} .
\end{aligned}
$$

The number of $J_{n m}(n \neq m)$ entering (12) with a plus sign is equal to the number of $J_{n^{\prime} m^{\prime}} \quad\left(n^{\prime} \neq m^{\prime}\right)$ entering with a minus sign, and the same property concerns the number of $K_{n m}$ and $K_{n^{\prime} m^{\prime}}$ having opposite sign. Also because a single $J_{n_{e} n_{e}}$ entering (12) with a plus sign is combined with a single $J_{n_{g} n_{g}}$ having a minus sign, the electron excitation energy (12) is a fully convergent result also at $R \rightarrow 0$. In summary, for a thin potential tube, an excitation of some special kind of the electron pairs requires much lower energy than the one-electron excitations. In a limiting process of the cross-section of the potential tube tending to zero, any one-electron excitation requires an infinite quantity of energy, but this does not apply to a two-electron excitation of the kind discussed above. This property is used in the forthcoming sections of the paper.

\section{Positive Change of the Kinetic Energy and Negative Character of the Electron-Electron Interaction Change in Course of a Pair Excitation}

The change of the kinetic energy of the electron pair in course of transition from the level $n_{g}$ to the level $n_{e}$ is evidently a positive quantity, because $n_{e}>n_{g}$; see (10). But the change of the electron-electron interaction 
of the same pair for an excitation from $n_{g}$ to $n_{e}$ is coupled with a negative change of energy. For example, at small $R$ the Coulomb interaction energy of the pair located on level 1 is [17]

$$
\begin{aligned}
& J_{11}=-2 \frac{e^{2}}{L} A_{0,2} \\
& +\frac{e^{2}}{L}(-3 \ln R+3 \ln L+3 \ln 2-\ln \pi+3 \beta-\ln 2-\gamma)
\end{aligned}
$$

whereas the same interaction energy on level 2 is

$$
\begin{aligned}
& J_{22}=-2 \frac{e^{2}}{L} A_{0,4} \\
& +\frac{e^{2}}{L}(-3 \ln R+3 \ln L+3 \ln 2-\ln \pi+3 \beta-\ln 2-\gamma),
\end{aligned}
$$

where $\beta \cong 0.681$ is a term descending from the normalization process and $\gamma$ is the Euler constant. Since

$$
A_{0,2 n}=-\frac{2}{\pi} \operatorname{Si}(2 n \pi),
$$

where $\mathrm{Si}$ is the integral sinus, a difference of the Coulomb energy in level 2 and level 1 becomes a negative result:

$$
\begin{aligned}
& J_{22}-J_{11} \approx \frac{e^{2}}{L}\left[-\ln 2+2 \frac{2}{\pi}\left(-\frac{1}{4 \pi}+\frac{1}{2 \pi}\right)\right] \\
& =\frac{e^{2}}{L}\left(-\ln 2+\frac{1}{\pi^{2}}\right) \cong \frac{e^{2}}{L}(-0,59) .
\end{aligned}
$$

Here the integral sinus entering (15) has been calculated with a satisfactory accuracy from the formula

$$
\operatorname{Si}(x) \cong \frac{\pi}{2}-\frac{\cos x}{x}
$$

because $x$ equal to $\pi$ multiplied by an even integer number is a sufficiently large argument to apply the approximate formula (17).

A still more approximate approach to the change of the electron interaction part of the excitation energy of an electron pair excited from some level $n_{F}$, which can be considered as a Fermi level, to a level $n_{e}>n_{F}$, gives also a negative value:

$$
\Delta E_{e e} \approx \frac{e^{2}}{L}\left[-\ln \left(2 n_{e}\right)+\ln \left(2 n_{F}\right)\right] .
$$

For $n_{e}=2$ and $n_{F}=1$ this gives

$$
\Delta E_{e e} \approx-\frac{e^{2}}{L} \ln 2=-\frac{e^{2}}{L} 0.69
$$

instead of (16).

A superconducting character of a system requires an instability of the Fermi energy with respect to the pair excitation. This is attained for a full excitation energy

$$
\Delta E=\Delta E_{k i n}+\Delta E_{e e},
$$

where $\Delta E_{k i n}$ of (10) is added to $\Delta E_{e e}$ of (18), on condition $\Delta E_{e e}$ predominates over $\Delta E_{k i n}$.

With the aid of the term

$$
f=\frac{h^{2}}{8 m_{e} e^{2}}=0.026 \times 10^{-6} \mathrm{~cm}
$$

we can look for such $L=L_{c r}$ that

$$
\begin{aligned}
& \Delta E= \\
& \frac{h^{2}}{8 m_{e}}\left\{2\left(n_{e}^{2}-n_{F}^{2}\right) \frac{1}{L^{2}}+\frac{e^{2}}{L f}\left[-\ln \left(n_{e}\right)+\ln \left(n_{F}\right)\right]\right\}=0 .
\end{aligned}
$$

This gives for $L=L_{c r}$ the equation

$$
L_{c r}=2 \frac{n_{e}^{2}-n_{F}^{2}}{\ln \left(n_{e}\right)-\ln \left(n_{F}\right)} f
$$

where

$$
n_{e}=n_{F}+\Delta n
$$

and $\Delta n>0$. In order to give a negative excitation energy of the electron pair the length $L$ of the potential tube should be larger than some critical length $L_{c r}$ calculated in (22). In Figure 2(a) we plot $L_{c r}$ as a function of $n_{F}$ for three integer values of $\Delta n$.

Here it should be noted that in calculating $L_{c r}$ in (22) the electron interaction of the 'bare' pair submitted to an excitation with the electron pairs occupying deeper levels in the Fermi sea has been neglected. The correction due to that interaction effect is calculated in Section 4.

\section{Pair Interaction with the Fermi Sea}

Section 3 examined only the Coulomb electrostatic energy of a 'bare' electron pair; no exchange energy is then involved because electrons have an opposite spin. In reality, such a pair is interacting with the Fermi sea. Our aim is to calculate a correcting term equal to the change of the pair interaction due to the presence of other electrons in the gas in course of an excitement of the electron pair. The index of an excited level of the pair is assumed to be $n_{F}+1$, and the level on which the pair is located before excitation let be $n_{F}$; see Figure 1. The interaction energy of the pair located in $n_{F}+1$ with the Fermi sea is equal to

$$
2 \sum_{i=1}^{i=n_{F}-1}\left(2 J_{i, n_{F}+1}-K_{i, n_{F}+1}\right)
$$

(the level $n_{F}$ is emptied), whereas a similar interaction energy of the pair located in the level $n_{F}$ is 


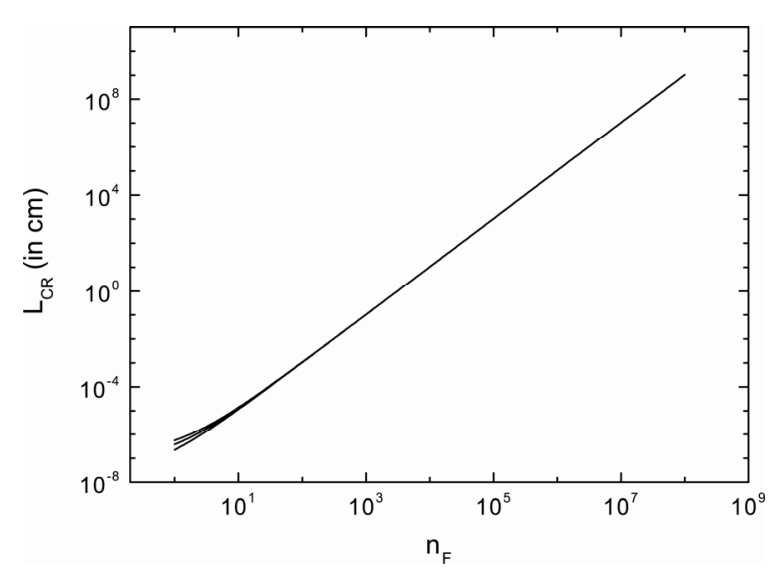

(a)

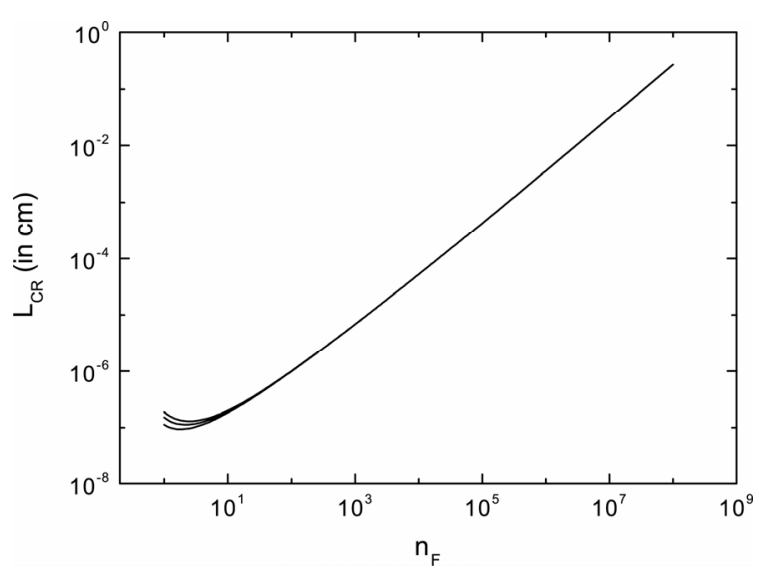

(b)

Figure 2. Critical length $L_{c r}$ of the electron gas potential tube. A formation of the gap at the Fermi level requires the tube length $L>L_{c r}$. (a) $L_{c r}$ for an excitation of a single electron pair with neglected pair interaction with the Fermi sea [see (22)]; (b) $L_{c r}$ calculated for an excited electron pair interacting with the Fermi sea [see (32)]. The curves are plotted for $\Delta n=1$ (lowest $\left.L_{c r}\right), 2,3$ (highest $L_{c r}$ ).

$$
2 \sum_{i=1}^{i=n_{F}-1}\left(2 J_{i, n_{F}}-K_{i, n_{F}}\right) .
$$

The factor of 2 before the integral $J$ within the brackets is due to the fact that the Coulomb energy concerns an interaction which involves both kinds of spin, whereas the exchange integral concerns an interaction only between electrons having spin of the same kind [16].

In effect, the correcting term representing the change of the electron interaction energy connected with the pair transition from the level $n_{F}$ to the level $n_{F}+1$ becomes

$$
\Delta E_{\text {int }}^{\text {corr }}=4 \sum_{i=1}^{i=n_{F}-1}\left(J_{i, n_{F}+1}-J_{i, n_{F}}\right)-2 \sum_{i=1}^{i=n_{F}-1}\left(K_{i, n_{F}+1}-K_{i, n_{F}}\right) .
$$

For large $n_{F}$ the first sum in (26), which can be approximated by [17]

$$
\sum_{i=1}^{i=n_{F}-1}\left(J_{i, n_{F}+1}-J_{i, n_{F}}\right) \approx-\frac{1}{2 n_{F}} \frac{e^{2}}{L},
$$

becomes negligibly small in comparison with the second sum in (26) which becomes approximately equal to [17]:

$$
\sum_{i=1}^{i=n_{F}-1}\left(K_{i, n_{F}+1}-K_{i, n_{F}}\right) \approx \ln \left(2 n_{F}\right) \frac{e^{2}}{L} .
$$

In total, the correction of energy due to the pair interaction with the Fermi sea calculated from (27) and (28) is a negative term:

$$
\Delta E_{e e}^{c o r r} \cong-2 \ln \left(2 n_{F}\right) \frac{e^{2}}{L} .
$$

Evidently, for large $n_{F}$, the excitation energy of a 'bare' electron pair calculated for $n_{e}=n_{F}+1$ [see (18) and (18a) for a special case of $n_{e}=2$ and $\left.n_{F}=1\right]$ is negligibly small with respect to $\Delta E_{e e}^{\text {corr }}$, so

$$
\Delta E_{e e} \approx \Delta E_{e e}^{c o r r}=-2 \ln \left(2 n_{F}\right) \frac{e^{2}}{L} \Delta n .
$$

This is a negative term because the difference of the exchange integrals entering (26) has a minus sign. The interval $\Delta n$ is that given in (23), where $\Delta n>1$ can be also admitted.

Consequently, a critical length of the potential tube descending from the requirement that

$$
\Delta E=\Delta E_{k i n}+\Delta E_{e e}=0
$$

[see (22)] becomes

$$
L_{c r} \cong \frac{2\left(n_{e}^{2}-n_{F}^{2}\right)}{2 \Delta n \ln \left(2 n_{F}\right)} f=\frac{2 n_{F}}{\ln \left(2 n_{F}\right)} f .
$$

In the formula for $L_{c r}$ the number $n_{F} \gg 1$ is assumed. The length $L_{c r}$ is plotted in Figure 2(b) as a function of $n_{F}$ for three values of $\Delta n: 1,2$, and 3 .

\section{Energy Gap at the Fermi Level and the Coherence Length}

At length $L=L_{\text {ext }}=2 L_{c r} \quad$ [see (32)] the difference $\Delta E$ entering (31) plotted as a function of $L$ attains its minimum; see Figure 3.

For $L>L_{e x t}$ there exists a slow increase of $\Delta E$ to the value $\Delta E \rightarrow 0$ attained at very large $L$. The absolute value of $\Delta E$ at $L=L_{e x t}$ can be considered as 
the energy gap for the pair excitation spectrum. This is:

$$
E_{g}=\left|\Delta E_{e e}\right|=|\Delta E|_{L=L_{e x t}}=\frac{m_{e} e^{4}}{h^{2}} \frac{2\left[\ln \left(2 n_{F}\right)\right]^{2} \Delta n}{n_{F}} \text {. }
$$

A plot of $E_{g}$ versus $n_{F}$ is presented in Figure 4.

The energy $E_{g}$ in (33) can be referred to a distance $\xi_{0}$ called the coherence length [18,19]:

$$
\xi_{0}=\frac{2 \hbar v_{F}}{\pi E_{g}} .
$$

The velocity $v_{F}$ at the Fermi level is estimated as equal to

$$
v_{F}=\frac{n_{F} h}{2 m_{e} L},
$$

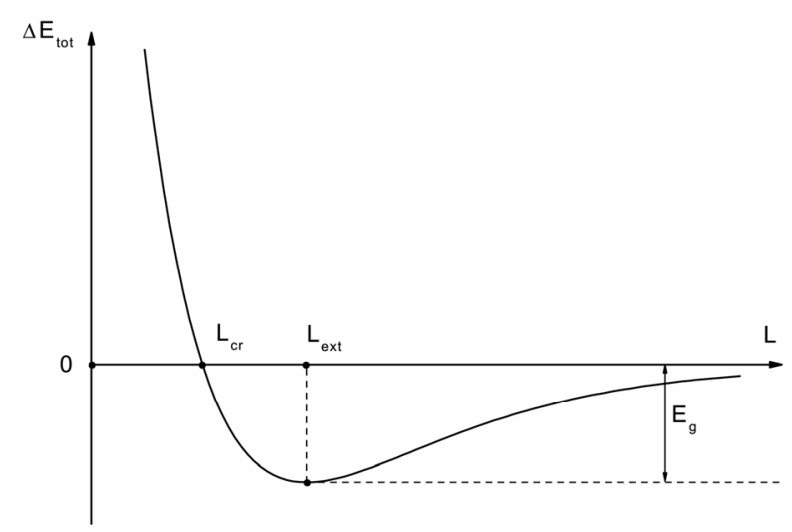

Figure 3. The excitation energy $\Delta E$ of the electron pair [see (31)] in a one-dimensional non-magnetic electron gas plotted versus the length $L$ of the gas potential tube. The minimum position $L_{e x t}$ is attained at $2 L_{c r}$, where $L_{c r}$ is a critical length at which $\Delta E=0$; see (32).

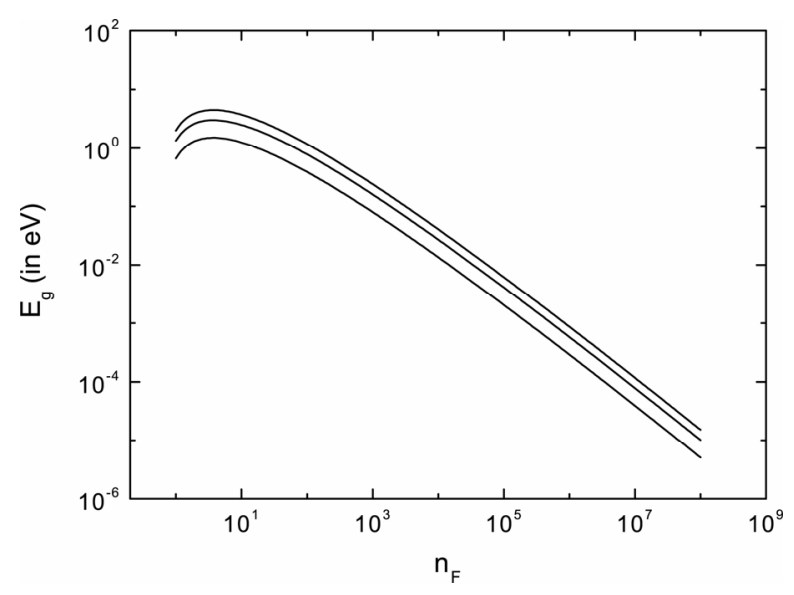

Figure 4. Energy gap $E_{g}$ (in $\mathrm{eV}$ ) for the pair excitation of a non-magnetic gas [see (33) and (30)]. The lowest $E_{g}$ is for $\Delta n=1$, the highest one-for $\Delta n=3$. since $v_{F}$ satisfies the following equation for the Fermi energy of the non-interacting gas:

$$
E_{F}=\frac{n_{F}^{2} h^{2}}{8 m_{e} L^{2}}=m_{e} \frac{v_{F}^{2}}{2} .
$$

The formula (34) combined with (35) for $L=L_{\text {ext }}=2 L_{c r}$ taken from (32) gives

$$
\xi_{0} \cong \frac{1}{2 \pi^{2}} \frac{h^{2}}{m_{e} e^{2}} \frac{n_{F}}{\Delta n \ln \left(2 n_{F}\right)}
$$

where

$$
\frac{h^{2}}{m_{e} e^{2}}=8 f \cong 0.209 \times 10^{-6} \mathrm{~cm} .
$$

A plot of the function $\xi_{0}\left(n_{F}\right)$ for several $\Delta n=n_{e}-n_{F}$ is done in Figure 5.

\section{Relation between the Critical Length $L_{c r}$ and the Coherence Length $\xi_{0}$}

These parameters can be compared together giving an especially simple relation, on condition an excitation of the electron gas having large $n_{F}$ is considered. Simultaneously, the change $\Delta n$ of $n_{F}$ is assumed to be a relatively small number. In this case, because of (32), (37) and (38), we obtain the ratio:

$$
\frac{\xi_{0}}{L_{c r}}=\frac{2}{\pi^{2}} \frac{1}{\Delta n}
$$

which means a proportionality between $L_{c r}$ and $\xi_{0}$, with the factor of proportionality dependent on $\Delta n$.

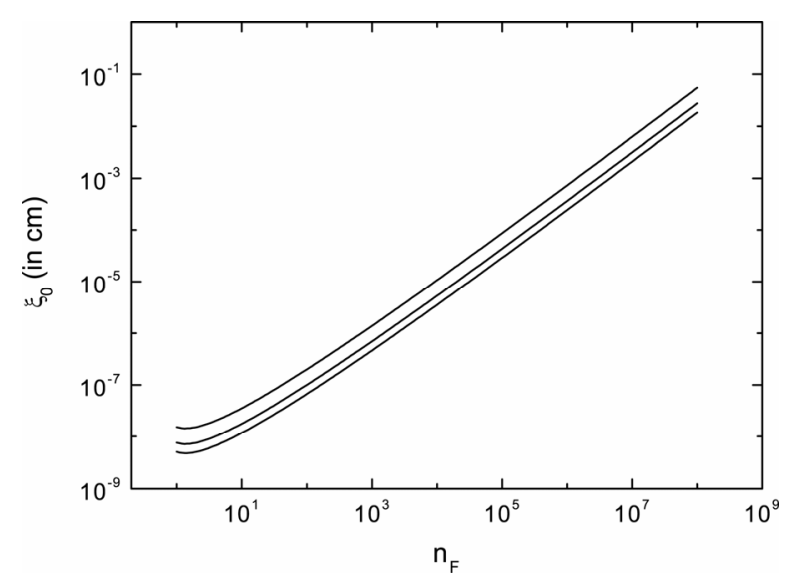

Figure 5. Coherence length $\xi_{0}$ [see (37)] plotted for several pair transitions $\Delta \boldsymbol{n}=\boldsymbol{n}_{\boldsymbol{e}}-\boldsymbol{n}_{\boldsymbol{F}}$ as a function of $n_{F}$. The lowest $\xi_{0}$ is for $\Delta \boldsymbol{n}=\mathbf{3}$, the highest one - for $\Delta \boldsymbol{n}=\mathbf{1}$; see also (39) and Figure 2. 


\section{Effect of the Magnetic Field on a Superconducting Behaviour of a One-Dimensional Many-Electron System}

A well-known property of three-dimensional superconductors is a destruction of the superconducting state due to an external magnetic field. A similar property can be expected in a one-dimensional system. In this case the magnetic field can do a reversal of the electron spin at least at some levels near the Fermi level $n_{F}$. In effect, the excitations of electron pairs near that level are stopped and the low-energy one-electron transitions, absent in a non-magnetic gas case, become then possible. An example of the pattern of levels of this kind is presented in Figure 6.

The transition energy of a single electron between level $n_{F}$ and $n_{F}+1$ becomes a finite, i.e. convergent, result at $R \rightarrow 0$ in this case. Assuming the electronelectron interaction as only important part in the excitation energy, and the kinetic part of that energy considering as negligible, the transition energy from the level $n_{F}$ to the level $n_{e}>n_{F}$ becomes:

$$
\begin{aligned}
& \Delta E \approx \Delta E_{e e}^{\text {paramagnetic }}=\sum_{i=1}^{i=n_{F}-1}\left(J_{n_{e}, i}-K_{n_{e}, i}\right) \\
& -\sum_{i=1}^{i=n_{F}-1}\left(J_{n_{F}, i}-K_{n_{F}, i}\right)+\sum_{i=1}^{i=n_{F}-2}\left(J_{n_{e}, i}-J_{n_{F}, i}\right) .
\end{aligned}
$$

The first two sums on the right-hand side of (40) come from the interaction between electrons having the same kind of spin, the last sum is due to the interaction between electrons having opposite spin. Approximately, the expression (40) becomes [17]

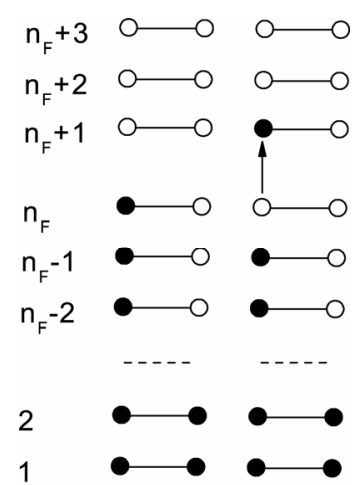

(a)

(b)

Figure 6. Pattern of the energy levels filling the Fermi sea of a partly magnetized electron gas: (a) a scheme before excitation; (b) a scheme after a single-electron excitation at the Fermi level. Full circles denote the occupied electron states.

$$
\begin{aligned}
& \Delta E_{e e}^{\text {paramagnetic }} \cong-\frac{n_{e}-\left(n_{F}-1\right)}{2\left(n_{F}-1\right)} \frac{e^{2}}{L} \\
& -\left(n_{e}-n_{F}\right) \ln \left[2\left(n_{F}-1\right)\right] \frac{e^{2}}{L}-\frac{n_{e}-\left(n_{F}-2\right)}{2\left(n_{F}-2\right)} \frac{e^{2}}{L} \\
& \approx-\Delta n \ln \left(2 n_{F}\right) \frac{e^{2}}{L} .
\end{aligned}
$$

The end result in (40a) holds on condition

$$
\Delta n=n_{e}-n_{F} \ll n_{F},
$$

and $n_{F}$ is assumed to be a large number.

The density of energy due to the presence of the magnetic field in a sample can be referred to the energy difference between the paramagnetic and non-magnetic state. This is expressed by the formula (see e.g. [20,21])

$$
\begin{aligned}
& 18 \pi H_{c}^{2}=\frac{E^{\text {paramagnetic }}-E^{\text {non-magnetic }}}{\Omega} \\
& \cong \frac{\Delta E_{e e}^{\text {paramagnetic }}-\Delta E_{e e}^{\text {non-magnetic }}}{\Omega}
\end{aligned}
$$

where $\Omega$ is the volume of the metal sample:

$$
\Omega=\pi R^{2} L .
$$

The energy difference in (42) can be limited to a difference of the electron excitation energies near the Fermi level. Because of (40a) and the relation

$$
\Delta E_{e e}^{n o n-m a g n e t i c}=\Delta E_{e e}=-2 \Delta n \ln \left(2 n_{F}\right) \frac{e^{2}}{L}
$$

[see (30)], the formula (42) becomes

$$
\begin{aligned}
& 18 \pi H_{c}^{2}=\frac{\left[-\Delta n \ln \left(2 n_{F}\right)+2 \Delta n \ln \left(2 n_{F}\right)\right]}{\Omega} \frac{e^{2}}{L} \\
& =\frac{\Delta n \ln \left(2 n_{F}\right)}{\Omega} \frac{e^{2}}{L} .
\end{aligned}
$$

The expression (45) is evidently a positive result for any $\Delta n>0$.

The size of $H_{c}$ can be expressed in terms of parameters $R$ and $L$ entering the right hand side of (45). For physical reasons a constant

$$
R \cong 2 \times 10^{-8} \mathrm{~cm}
$$

can be put equal roughly to the length of the order of an atomic radius estimated for the atoms entering the whole of the atomic chain forming the core of a one-dimensional system [22]. On the other hand, $L$ should be not smaller than $L_{c r}$ calculated in Section 4. A plot of $H_{c}$ versus $n_{F}$ is done in Figure 7 (see the singlet (s) curve).

\section{Penetration Depth in One-Dimensional Superconductors and Its Kinds}

With the action of the magnetic field on a supercon- 


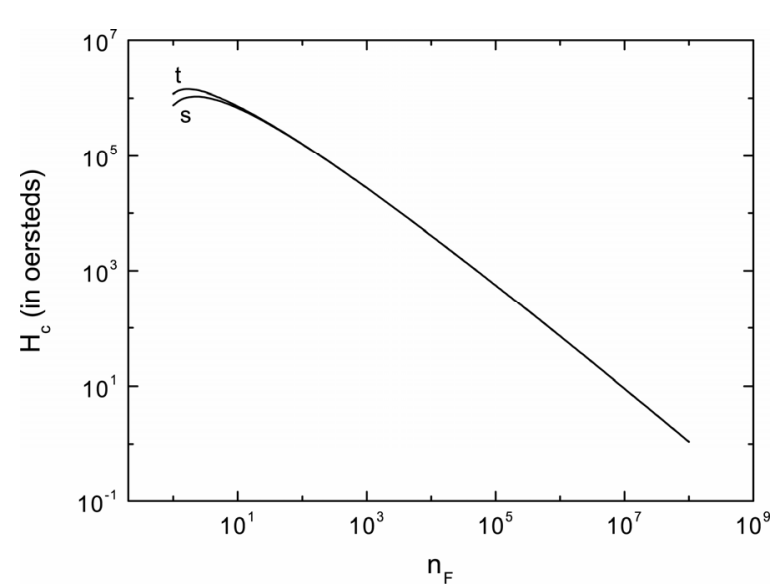

Figure 7. Critical magnetic field $H_{c}$ (in oersteds) calculated from a difference of the energy density between the paramagnetic and non-magnetic electron gas; see (45) for the singlet states (curve $s$ ); for the triplet states see (62) (curve $t$ ).

ductor is associated the penetration depth $\lambda$. If the field outside a superconductor is equal to $H_{0}$, its decrease measured inside a sample on a distance $z$ from its surface is represented by the formula $[23,24]$

$$
H(z)=H_{0} e^{-z / \lambda}
$$

where

$$
\lambda=\left(\frac{m_{e} c^{2}}{4 \pi n_{\text {tot }} e^{2}}\right)^{1 / 2}
$$

and

$$
n_{\text {tot }}=\frac{2 n_{F}}{\pi R^{2} L}
$$

is a formula for the electron density within a quasi onedimensional sample, valid on condition $n_{F}$ is a large number. This $\lambda$ is independent of the strength of the magnetic field.

However, there exists also another penetration depth $\lambda^{I I}$ which can be associated with the coherence length. This parameter is useful in classifying the superconductors, namely those belonging to the second kind of superconductors. According to the Ginzburg-Landau theory, the free energy of a superconductor of the second kind can be defined with the aid of two parameters, $\alpha$ and $\beta$ [23]. The first parameter is coupled with the coherence length $\xi_{0}$ with the aid of the equation

$$
|\alpha|=\frac{\hbar^{2}}{2 m_{e} \xi_{0}^{2}},
$$

whereas the second parameter is defined by a critical magnetic field $H_{c}$ and $\alpha$ :

$$
\beta=\frac{4 \pi \alpha^{2}}{H_{c}^{2}} .
$$

If we put

$$
\rho_{\text {part }}=\frac{|\alpha|}{\beta},
$$

the penetration length $\lambda^{I I}$ becomes

$$
\lambda^{I I}=\left(\frac{m_{e} c^{2}}{4 \pi \rho_{\text {part }} e^{2}}\right)^{1 / 2} .
$$

In this formula $\rho_{\text {part }}$ replaces $n_{\text {tot }}$ present in (47a). A comparison of $\lambda^{I I}$ and $\xi_{0}$, important in classifying the properties of superconductors, is discussed below; Section 9.

A plot of $\lambda$ calculated from (47a) is done in Figure $\mathbf{8}$, a similar plot of $\lambda^{I I}$ obtained from (51) is presented in Figure 9, curve $s$. This dependence concerns a singlet excited state of the electron pair.

\section{Singlet-Singlet and Triplet-Triplet Excitations of the One-Dimensional Electron Gas}

Transitions of the electron pairs in a non-magnetic gas discussed in Section 3 and Section 4 concerned the electrons of opposite spin forming the singlet states; see e.g. [16]. But a situation may exist when an electron pair of the same spin is located near the Fermi level, for example on the levels $n_{F}$ and $n_{F}+1$; see Figure 10 . This situation should be classified as a triplet state. In the next step, one of electrons of the pair can be promoted from $n_{F}+1$ to a level $n_{F}+q$, where $q>1$, leaving the electron on $n_{F}$ unchanged:

$$
\left(n_{F}, n_{F}+1\right) \rightarrow\left(n_{F}, n_{F}+q\right) .
$$

The electron-electron interaction energy of the pair in its ground state $\left(n_{F}, n_{F}+1\right)$ is equal to

$$
E_{e e}^{\text {pair }}\left(n_{F}, n_{F}+1\right)=J_{n_{F}, n_{F}+1}-K_{n_{F}, n_{F}+1},
$$

on condition the interaction with the remainder of electrons in the gas is neglected. A convergent result is attained for (53) at $R \rightarrow 0$, because the $\ln R$ divergencies entering $J$ and $K$ cancel together. But the same property concerns also the interaction energy of an excited pair, say that obtained when one of electrons is promoted from the level $n_{F}+1$ to some level $n_{F}+q$ :

$$
E_{e e}^{p a i r}\left(n_{F}+q, n_{F}\right)=J_{n_{F}+q, n_{F}}-K_{n_{F}+q, n_{F}} .
$$

Evidently, a difference of two convergent energies in (54) and (53), which is the change of the electron- 


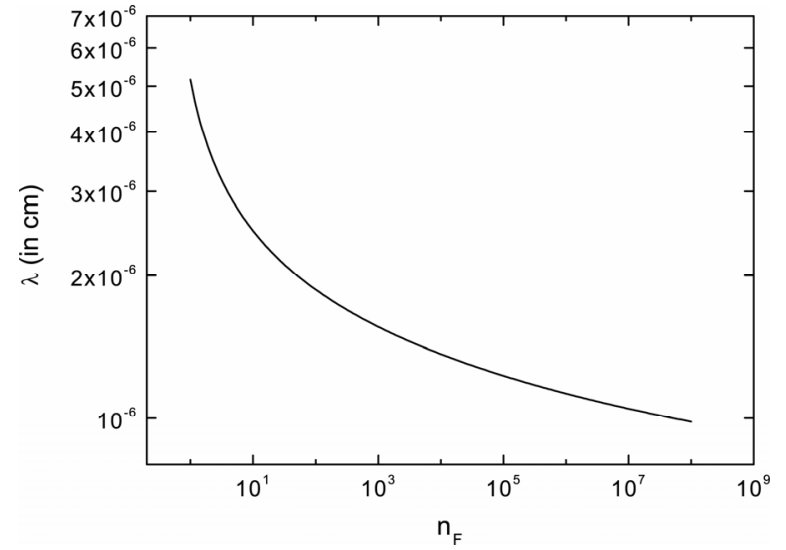

Figure 8. Penetration depth $\lambda$ of the magnetic field calculated from (47a) is the same for the singlet and triplet states; see (57).

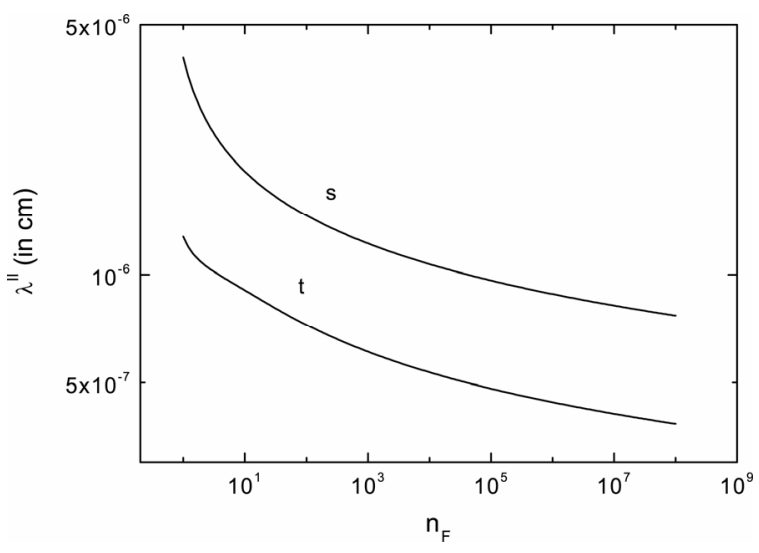

Figure 9. Penetration depth $\lambda^{I I}$ characteristic for a superconductor of the second kind calculated for the singlet and triplet states [see (51)]. $H_{c}$ and $\xi_{0}$ are different for singlets and triplets; see (61) and Figure 7.

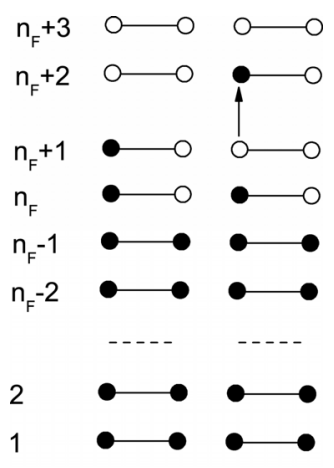

(a)

(b)

Figure 10. Pattern of the energy levels in a one-dimensional electron gas having near the Fermi level an electron pair in the triplet state. (a) the gas before an excitation of the pair, (b) the gas having an excited triplet pair. electron interaction energy due to the transition between $n_{F}+1$ and $n_{F}+q$, is also a convergent result at $R \rightarrow 0$.

A full change of the electron-electron interaction energy in a triplet state associated with a one-electron transition from the level $n_{F}+1$ to the level $n_{F}+q$ is obtained when the interaction energy of the pair with other electrons in the gas is taken into account. This calculation replaces the difference between energies in (54) and (53) by the change of the interaction energy similar to that given in (40) and (40a):

$$
\Delta E_{e e}^{\text {triplet }}=\sum_{i=1}^{i=n_{F}}\left[2\left(J_{n_{F}+q, i}-J_{n_{F}+1, i}\right)-\left(K_{n_{F}+q, i}-K_{n_{F}+1, i}\right)\right] .
$$

The first sum in brackets represents a contribution of the Coulomb integrals. This can be omitted at $R \rightarrow 0$ in comparison with the second sum in (55) which is due to the exchange integrals. A full excitation energy is approximately equal to:

$$
\Delta E_{e e}^{\text {triplet }} \approx-(q-1) \ln \left(2 n_{F}\right) \frac{e^{2}}{L}
$$

The result in (56) is a negative number on condition $q>1$.

The critical length $L_{c r}$ for the triplet states can be obtained on the basis of (56), for example for $q=2$. In this case we have a promotion of an electron from $n_{F}+1$ to $n_{F}+2$. Because of (56) the formula which replaces (32) becomes

$$
\begin{aligned}
& L_{c r}^{\text {triplet }}=\frac{\left(n_{F}+2\right)^{2}-\left(n_{F}+1\right)^{2}}{\ln \left(2 n_{F}\right)} f \\
& \cong \frac{2 n_{F}}{\ln \left(2 n_{F}\right)} f=L_{c r},
\end{aligned}
$$

so $L_{c r}$ in the triplet and singlet state remain approximately the same.

Another situation is for $\xi_{0}$. A comparison of $\Delta E_{e e}^{\text {triplet }}$ in (56) calculated for $q=2$, so

$$
\Delta n=n_{F}+q-\left(n_{F}+1\right)=q-1=1,
$$

with

$$
\Delta E_{e e}^{\text {singlet }}=\Delta E^{\text {non-magnetic }}=-2 \ln \left(2 n_{F}\right) \frac{e^{2}}{L}
$$

calculated in (30) for a singlet transitions having $\Delta n=1$, shows that for the same $\Delta n$ the lowering of the excitation energy due to the electron-electron interaction calculated in a triplet state is only a half of the result obtained for a singlet state. Consequently to (33), a reduction of the energy gap for the triplet-triplet transitions is obtained to the value 


$$
E_{g}^{\text {triplet }}=\frac{1}{2} E_{g}^{\text {singlet }}=\frac{1}{2} E_{g},
$$

where $E_{g}$ is that given (33). In effect, the coherence length

$$
\xi_{0}^{\text {triplet }}=2 \xi_{0} \equiv 2 \xi_{0}^{\text {singlet }},
$$

because the formula in (34) can be applied also for $\xi_{0}^{\text {triplet }}$. The results obtained in (57) and (61) indicate that the ratio $\xi_{0} / L_{c r}$ calculated in (39) becomes for the triplet transitions twice as large as that obtained for the singlet transitions.

An estimate of the critical magnetic field for the triplet states takes into account the fact that the electronelectron interaction energy near the Fermi level is of a paramagnetic character, so it does not vary much upon the action of an external magnetic field. In effect, the expense of the field energy is expected to be done mainly in order to change the kinetic energy:

$$
\frac{1}{8 \pi} H_{c}^{2}=\frac{\Delta E}{\Omega}=\frac{h^{2}}{8 m_{e} L^{2}} \frac{\left(n_{F}+2\right)^{2}-\left(n_{F}+1\right)^{2}}{\Omega} .
$$

Here $L=L_{c r}$ from (57) has to be substituted into (62), as well as in $\Omega$ in (43). A plot of $H_{c}$ of this kind done versus $n_{F}$ is given in Figure 7. This plot is there compared with that done for the singlet states obtained with the aid of the formula (45). In the next step, $\xi_{0}^{\text {triplet }}$ of (61) and $H_{c}$ of (62) can be applied to the calculation of $\lambda^{I I}$ for the triplet states following the formulae (48)-(51); see Figure 9. In Figure 11 we compare the plots of $n_{\text {tot }}$ entering (47a) and (47b) with $\rho_{\text {part }}$ obtained for singlet and triplet transitions calculated from (48)-(50).

\section{Critical Current and Critical Temperature for One-Dimensional Systems}

A critical current for destruction of the superconducting state can be obtained from the ratio (see e.g. [19,25]):

$$
J_{c} \approx \frac{H_{c}}{\lambda}
$$

where $H_{c}$ is a critical magnetic field and $\lambda$ is a penetration depth. We can calculate $J_{c}$ separately for the singlet and triplet states. The $\lambda$ of two kinds, that of (47a) as well as $\lambda^{I I}$ of (51) can be applied. The plots of the dependencies of $J_{c}$ in (63) on $n_{F}$ are presented in Figure 12.

The size of a critical temperature $T_{c}$ can be estimated from $E_{g}$ assuming the approximate formula:

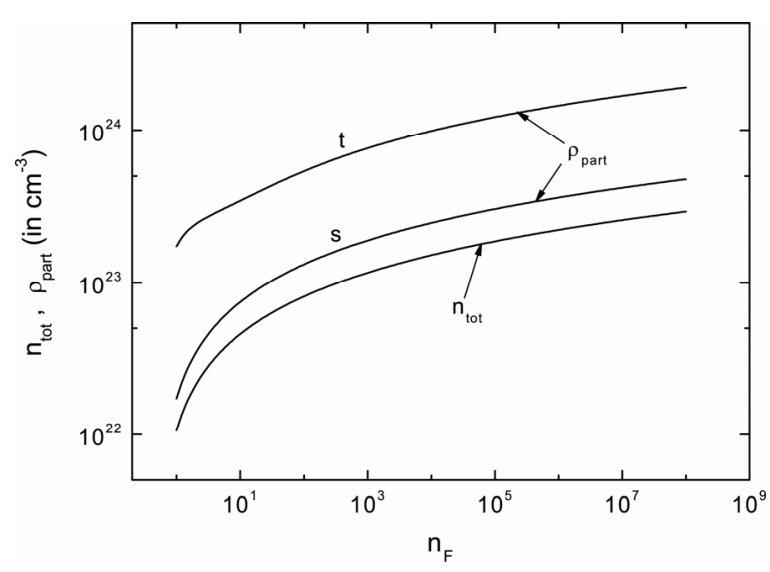

Figure 11. Electron gas density $n_{\text {tot }}$ in the potential tube [see (47b)] compared with the particle density $\rho_{\text {part }}$ entering the calculation of $\lambda^{I I}$ for (a) the case of the singlet states ( $s$ ), (b) the triplet states ( $t)$; see (50). A characteristic point is that $n_{t o t}$ is not essentially different from $\rho_{\text {part }}$.

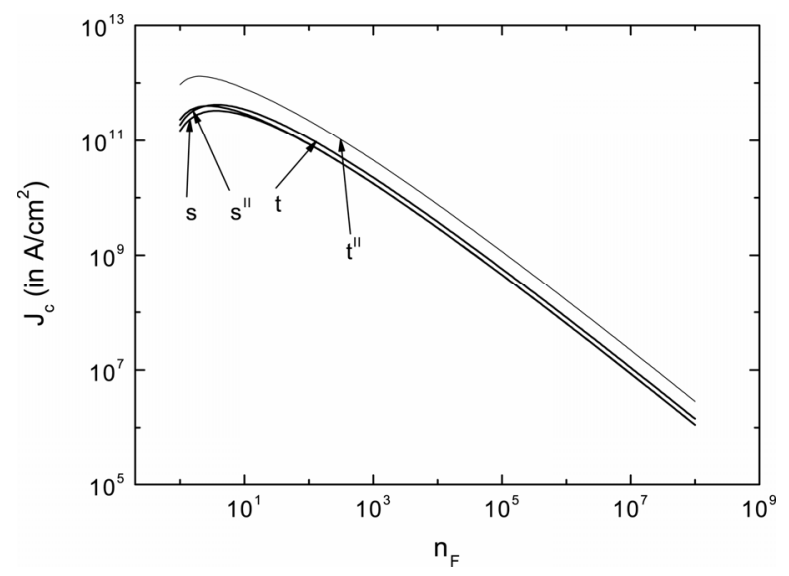

Figure 12. Critical current intensity $J_{c}$ for the onedimensional superconductors calculated as a function of $n_{F}$; see (63). 1 Oersted $\approx 0.8 \mathrm{~A} / \mathrm{cm}$. The curves with the indices $s$ and $s^{I I}$ referring to the singlet states are calculated for $H_{c}$ from (45) and $\lambda, \lambda^{I I}$ taken from (47a), (51), respectively; the curves with the indices $t$ and $t^{I I}$ referring to the triplet states are calculated for $H_{c}$ from (62) and $\lambda, \lambda^{I I}$ indicated above. For large $n_{F}$ the curve $s$ merges with $s^{I I}$.

$$
T_{c} \approx \frac{E_{g}}{k_{B}}
$$

where $k_{B}$ is the Boltzmann constant. The plots of $T_{c}$ are done on Figure 13. 


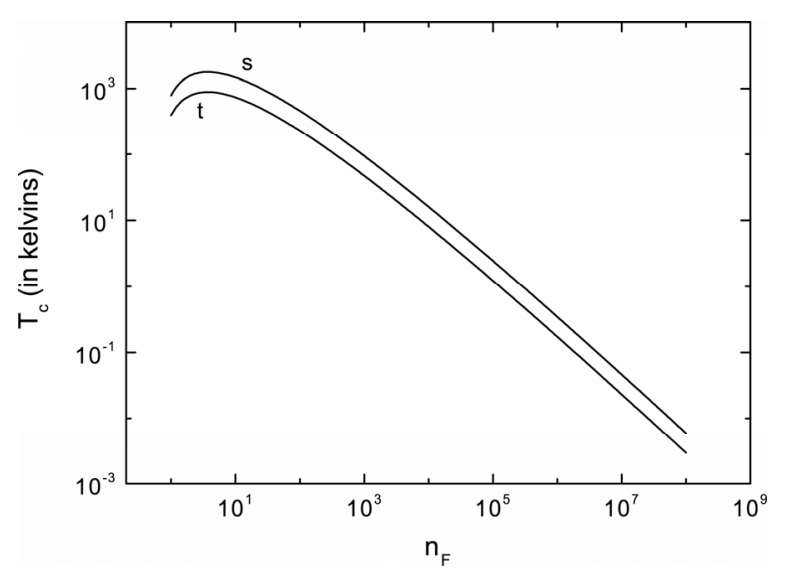

Figure 13. Critical temperature $T_{c}$ calculated from (64) as a function of $n_{F}$. Curve $s$ : temperature $T_{c}$ for singlets; curve $t$ : temperature $T_{c}$ for triplets.

\section{Summary}

An attempt is done to approach the problem of superconductivity in quasi one-dimensional systems basing mainly on an analysis of the electron-electron interaction and kinetic energy effects; an important problem of the many-electron wave function is neglected here.

A well-known fact is that the geometry of the volume in which the electron charges are enclosed can influence considerably the interaction between them; see e.g. [26]. In particular, a one-dimensional volume provides us with the logarithmic divergencies in the interaction energy between the charges.

But in considering the superconductivity effects, the excitation energy of an electron system, and not the absolute value of the system energy, plays a dominant role. Consequently-in the first step-the excitation energy of a single electron pair, located in the vicinity of the Fermi level is considered. In an extremely thin potential tube the total energy of the pair tends to diverge. Nevertheless, this divergence is precisely cancelled in the case of a pair excitation. This holds on condition the pair occupies a single orbital quantum state, equally after and before the excitation process. This requirement implies that the absolute values of the electron momenta in the pair should be equal, but the spin states in that pair are opposite. Evidently, the presence of pairs of this kind leads to the singlet states.

An important property of the pair is that the Coulomb part of its excitation energy from a lower orbital quantum level to a higher level is a negative number. With a positive value of the kinetic excitation energy between the pair levels, we obtain a kind of competition between the changes of the electron-electron interaction and kinetic energies. Above some critical length of the potential tube, the interaction part predominates over the kinetic part, and the pair excitation is connected with a lowering of the energy of the system.

This elementary result obtained for a single electron pair can be extended to an excitation of the manyelectron system present in an almost one-dimensional gas volume. In particular, the interaction energy of the pair submitted to an excitation with the remainder of the gas electrons is taken into account. A characteristic point is that the size of the energy gap obtained when excitements of an isolated electron pair are only considered becomes much larger for the case of the pairs interacting with the Fermi sea. The critical length $L_{c r}$ for an isolated pair at large $n_{F}$ is also very large, but for an interacting gas $L_{c r}$ is reduced to about $10^{-2} \div 10^{-1} \mathrm{~cm}$.

Beyond of singlets, also excitements of the pairs representing the triplet states are taken into account. The parameters of the electron gas examined in the paper include the coherence length, critical magnetic field, critical current intensity and critical temperature. The plots of these parameters are done in dependence on the electron number present in the linear gas sample, which is approximately equal to $2 n_{F}$, where $n_{F}$ is the index representing the orbital quantum number at the Fermi level. For a macroscopic $n_{F} \approx 10^{8}$ we obtain: $E_{g}$ equal to about $10^{-5} \mathrm{eV}, \quad \xi_{0} \sim 10^{-2} \mathrm{~cm}, H_{c} \sim 1$ Oe, $\lambda \sim 10^{-6} \mathrm{~cm}, \quad \lambda_{\mathrm{s}}^{I I} \sim 10^{-6} \mathrm{~cm}$ and $\lambda_{t}^{I I} \sim 5 \times 10^{-7} \mathrm{~cm}$, $J_{c} \sim 10^{6} \mathrm{~A} / \mathrm{cm}^{2}$ for singlets and $10^{7} \mathrm{~A} / \mathrm{cm}^{2}$ for triplets, $T_{c} \sim 10^{-2} \mathrm{~K}$ for singlets and a half of that value for triplets.

\section{References}

[1] N. P. Butch, M. C. de Andrade and M. B. Maple, "Resource Letter Scy-3: Superconductivity,” American Journal of Physics, Vol. 76, No. 2, 2008, pp. 106-118.

[2] Y. P. Huang and D. W. Wang, "Quantum-Phase Diagrams of Fermionic Dipolar Gases in a Planar Array of One-Dimensional Tables,” Physical Review A, Vol. 80, No. 053610, 2009, p. 10.

[3] N. Belmechri, G. Abramovici and M. Herititier, "Phase Diagram and Critical Fields of Organic Quasi-1d Superconductors in an Applied Magnetic Field,” Physica B, Vol. 404, No. 19, 2009, pp. 3131-3133.

[4] K. Kajiwara, M. Tsuchiizu, Y. Suzumura and C. Bourbonnais, "Mechanism of the Singlet to Triplet Superconductivity Crossover in Quasi-One-Dimensional Organic Conductors," Journal of the Physical Society of Japan, Vol. 78, No. 104702, 2009, p. 11.

[5] D. Jerome, A. Mazaud, M. Ribault and K. Bechgaard, "Superconductivity in a Synthetic Organic Conductor (TMTSF) ${ }_{2} \mathrm{PF}_{6}$," Journal de Physique Lettres (Paris), 
Vol. 41, No. 4, 1980, pp. L95-L98.

[6] K. Bechgaard, C. J. Jacobsen, K. Mortensen, H. J. Pedersen and N. Thorup, "The Properies of Five Highly Conducting Salts: (TMTSF) ${ }_{2} \mathrm{X}, \mathrm{X}=\mathrm{PF}_{6}^{-}, \mathrm{AsF}_{6}^{-}, \mathrm{SbF}_{6}^{-}$, $\mathrm{BF}_{4}^{-}$and $\mathrm{NO}_{3}^{-}$, Derived from Tetramethyltetraselenafulvalene (TMTSF)," Solid State Communications, Vol. 33, No. 11, 1980, pp. 1119-1125.

[7] D. Jerome, "One Dimensional Organic Superconductors: beyond the Fermii Liquid Description,” Journal de Physique IV (Paris), Vol. 10, No. PR3, 2000, pp. 69-84.

[8] D. Jerome, “ $(\mathrm{TM})_{2} \mathrm{X}$ Organic Superconductors and Related Physics,” Molecular Crystals and Liquid Crystals, Vol. 380, No. 1, 2002, pp. 3-13.

[9] N. Dupuis, C. Bourbonnais and J. C. Nickel, "Superconductivity and Antiferromagnetism in Quasi-One-Dimensional Organic Conductors," Journal of Low Temperature Physics, Vol. 32, No. 4-5, 2006, pp. 380-391.

[10] W. A. Little, "Possibility of Synthesizing an Organic Superconductor," Physical Review, Vol. 134, No. 6A, 1964, pp. A1416-A1424.

[11] Y. Fuseya and M. Ogata, "Increase of Superconducting Correlation due to Dimensionality Change in Quasi-OneDimensional Conductors," Journal of the Physical Society of Japan, Vol. 76, No. 093071, 2007, p. 4.

[12] I. J. Lee, S. E. Brown and M. J. Naughton, "Unconventional Superconductivity in a Quasi-One-Dimensional System (TMTSF) ${ }_{2} \mathrm{X}$," Journal of the Physical Society of Japan, Vol. 75, No. 051011, 2006, p. 9.

[13] J. Friedel, "Quasi-Low-Dimensionality in a Weak Coupling Limit,” Physica C, Vol. 153-155, 1988, pp. 16101616.

[14] C. Bourbonnais and L. G. Caron, "New Mechanism of Phase Transitions in Quasi-One-Dimensional Conduc- tors,” Europhysics Letters, Vol. 5, No. 3, 1988, pp. 209215.

[15] H. Eyring, J. Walter and G. E. Kimball, “Quantum Chemistry,” Wiley, New York, 1957.

[16] C. C. J. Roothaan, "New Developments in Molecular Orbital Theory,” Reviews of Modern Physics, Vol. 23, No. 2, 1951, pp. 69-89.

[17] S. Olszewski, "Hartree-Fock Approximation for the OneDimensional Electron Gas,” Zeitschrift für Physik B, Vol. 45, No. 4, 1982, pp. 297-306.

[18] C. Kittel, “Quantum Theory of Solids,” 2nd Edition, Wiley, New York, 1987.

[19] M. Cyrot and R. Pavuna, "Introduction to Superconductivity and High- $T_{c}$ Materials," World Scientific, Singapore, 1992.

[20] N. H. March, W. H. Young and S. Sampanthar, "ManyBody Problem in Quantum Mechanics,” University Press, Cambridge, 1967.

[21] E. A. Lynton, "Superconductivity,” Methuen, London, 1962.

[22] E. Perfetto and J. Gonzalez, "Electronic Correlations of Small Diameter Carbon Nanotubes,” Journal of Physics: Condensed Matter, Vol. 18, No. 33, 2006, pp. s2105s2114.

[23] J. M. Ziman, "Principles of the Theory of Solids,” University Press, Cambridge, 1972.

[24] M. R. Schafroth, "Theoretical Aspects of Superconductivity,” In: F. Seitz and D. Turnbull, Eds., Solid State Physics, New York, 1960, pp. 293-498.

[25] C. Kittel, "Introduction to Solid State Physics," 7th Edition, Wiley, New York, 1996.

[26] D. Ivanenko and A. Sokolov, “Classical Field Theory,”(in Russian), GITTL, Moscow, 1949. 